\title{
A joint resource allocation method for multiple targets tracking in distributed MIMO radar systems
}

\author{
Xiyu Song ${ }^{*}$ (D), Nae Zheng, Shuhao Yan and Haiwen Li
}

\begin{abstract}
In order to simultaneously improve system performance and resource utilization of distributed multiple-input multiple-output (MIMO) radar systems, a joint resource allocation method is proposed to address the velocity estimation problem for multiple targets tracking in this paper. The paper focuses to improve the tracking performance for key targets using the remaining resources when the general targets have obtained resources to reach to tracking requirements. Firstly, a criterion minimizing the velocity estimation mean square error (MSE) for a key target is considered. Restricted by limited and relatively sufficient system resources and given velocity estimation requirements for general targets, a joint resource allocation optimization model with transmitters, receivers, transmitted power, and signal time is established. We propose a suboptimal method to approximately solve this problem. The method separates the optimization into three steps, where each step transforms the corresponding mixed-Boolean optimization problem into a second-order cone programming (SOCP) problem by convex relaxation. Finally, the approximately optimal solution can be obtained by cyclic minimization method. Extensive simulations indicate that compared with other methods, the proposed joint method can achieve the lowest velocity estimation MSE with the fewest transmitters. Meanwhile, limited by the given velocity estimation MSE, the proposed method can focus on the key target and achieve the whole velocity estimation error minimization while a greater flexibility for target tracking number can be obtained. Moreover, random experiments can further validate and evaluate the proposed method's effectiveness and traceability with the given scenario.
\end{abstract}

Keywords: Distributed MIMO radar systems, Multiple targets tracking, Antenna selection, Power allocation, Signal time allocation

\section{Introduction}

Distributed multiple-input multiple-output (MIMO) radar is a kind of multi-static radar, but it is different from the previous multi-static radar or netted radar [1, 2]. For the previous multi-static radar and netted radar system, each radar works independently, transmitting the local processing results to the CPU for information fusion. However, the distributed MIMO radar can fuse the echo information detected by each beam while the signal is being transmitted and received, which is equivalent to data fusion at the original signal level. MIMO radar systems can offer enhanced target detection and localization capability by spatial multichannel

\footnotetext{
* Correspondence: fsongxiyu@126.com

National Digital Switching System Engineering and Technological Research Center, Zhengzhou 450001, China
}

(c) The Author(s). 2018 Open Access This article is distributed under the terms of the Creative Commons Attribution 4.0 International License (http://creativecommons.org/licenses/by/4.0/), which permits unrestricted use, distribution, and reproduction in any medium, provided you give appropriate credit to the original author(s) and the source, provide a link to the Creative Commons license, and indicate if changes were made.

[3-5]. While distributed MIMO radar systems have advantages on the extended target model and multiple targets monitoring by viewing the targets from different angles [6]. Meanwhile, with the development of stealth technology, the distributed MIMO radar systems are widely studied for the detection of stealth targets. Therefore, distributed MIMO radar systems become the research hotspot in the field of radar technology. The advantages of distributed MIMO radar lies in its flexible signal design and optimized system structure. In fact, transmitted parameters and antenna configuration can be regarded as system resources. Therefore, the research of distributed MIMO radar should mainly focus on the problem of resource allocation. In practical application, the radar resources may be limited. For the better system performance, it is

\section{Springer Open}

the Creative Commons license, and indicate if changes were made. 
necessary to improve the resource utilization in the multiple tasks system [7-12]. In this case, the resource allocation problem of distributed MIMO radar becomes a hot topic, especially for the problem of power allocation in low probability of interception (LPI) system $[13,14]$. In the war of multiple target tracking [15], although target location is the mainly concerned parameter, target velocity estimation can have a significant effect on state prediction, which can further determine resource allocation results. Once the estimation error of target velocity is larger, especially for the target at a high speed, the predicted target state with estimation error will fail to obtain an optimal allocation result. Considering the variability and uncertainty of target velocity, the resource allocation problem of multiple targets velocity estimation of distributed MIMO radar is worth studying.

The existing researches usually regard Bayesian Cramer-Rao Bound (BCRB) $[16,17]$ as the parameter estimation metric for the moving target. In [17], the $\mathrm{BCRB}$ for the location and velocity estimation is derived, and the results indicate the target state estimation is affected by antenna selection and transmitted parameters. Resource allocation problem usually is regarding as a non-convex optimization model. Under the requirement of LPI, a sequential parameter convex approximation (SPCA) method is proposed to solve the non-convex power allocation problem of multiple targets localization in [18]. Considering that more antennas can lead to large data transmission and computation complexity, an antenna selection problem is analyzed in [19-21]. With given performance requirements, a heuristic method is employed to select the minimal antenna subset for lower computation complexity. For better performance, an efficient two-step semi-definite programming (SDP)-based solution is proposed in [22] to solve the problem of joint node selection and power allocation. And the non-convex optimization problem can also be transformed into a second-order cone programming (SOCP) problem [23]. Based on this, a joint method of antenna selection and power allocation for target localization is solved in [24].

However, the previous researches mainly focus to improve the overall system performance or resource utilization, but ignore the specific task demands. Therefore, there are still some deficiencies for the further study.

- In terms of targets, target state is complicated and changeable in wars, the velocity change needs to be controlled for better target state prediction. Subsequently, in the multiple targets tracking system, it is usually unreasonable to treat all the targets with the same requirement, radar system should set different tracking accuracy requirements for every target and divide them to different priorities according to system tasks, which has been ignored in [18].

- In terms of the system, existing researches usually just consider the system performance or resource utilization. While in the multiple targets tracking system, considering the demands of different priority targets, system performance and resource utilization can be improved at the same time. Moreover, more receivers can increase communication load and computation complexity, which has been ignored in [24].

- In terms of resource allocation method, SPCA method proposed in [18] can just reach to a local convergence, while SOCP can reach to an approximate global convergence in [15]. To maximize the utilization, a joint multiple resources allocation method will contribute to better effects.

In this paper, we develop a resource allocation method for the better velocity estimation. Targets are divided into general targets and key targets according to different velocity accuracy requirements. Supposing that the system resources are relatively sufficient, the optimization object is to improve the estimation performance for key targets using all the remaining resources when general targets have met the given velocity estimation mean square error (MSE). In this paper, limited system resources and partial given performance requirements are considered. In this case, resource utilization and system performance can be improved together. To exploit system potential, a joint resource allocation problem including transmitter, receiver, transmitted power, and signal time, is studied in this work, and the corresponding optimization problem is solved by SOCP via convex relaxation. Finally, effectiveness of proposed method is verified by extensive simulations.

The paper is organized as follows. In Section 2, we introduce the system model and performance metric for velocity estimation. In Section 3, we proposed a three-step SOCP optimization method for the resource allocation model. In Section 4, the performance of the proposed method is evaluated by simulations where fixed and random distributed system layouts are respectively discussed. Finally, conclusions are drawn in Section 5 .

\section{System model and performance metric}

In this section, we introduce the system state model, observation model, and present the performance 
metric for multiple targets velocity estimation in distributed MIMO radar systems.

\subsection{State model}

Consider a distributed MIMO system consisting of $M$ transmitters located at $\left(x_{m}^{\mathrm{t}}, y_{m}^{\mathrm{t}}\right), m=1, \ldots, M$, and $N$ receivers located at $\left(x_{n}^{\mathrm{r}}, y_{n}^{\mathrm{r}}\right), n=1, \ldots, N$. There are $Q$ moving targets of state $\left(x^{q}, y^{q}, v_{x}^{q}, v_{y}^{q}\right), q=1, \ldots, Q$. Orthogonal waveforms are transmitted with a low pass equivalent signal $s_{m}(t), m=1, \ldots, M$. The transmitted power vector is $\mathbf{p}=\left[p_{1}, \ldots, p_{m}, \ldots, p_{M}\right]^{\mathrm{T}}$. The signal time vector is $\mathbf{t}=\left[t_{1}, \ldots, t_{m}, \ldots, t_{M}\right]^{\mathrm{T}}$, where $p_{m}$ and $t_{m}$ respectively represent the transmitted power and the signal duration time for the $m$ th transmitter.

Define the target state vector $\mathbf{x}_{k}^{q}=\left[x_{k}^{q}, y_{k}^{q}, v_{x, k}^{q}, v_{y, k}^{q}\right]^{\mathrm{T}}$ for the qth target at state $k$. The target motion model can be expressed as

$$
\mathbf{x}_{k+1}^{q}=\mathbf{F x}_{k}^{q}+\mathbf{v}_{k}^{q}
$$

where $\mathbf{F}$ is state transition matrix. $\mathbf{v}_{k}^{q}$ is zero-mean and white Gaussian noise with covariance matrix $\mathbf{Q}_{k}$. For an uniform motion model, $\mathbf{F}$ and $\mathbf{Q}_{k}$ are of the form (2) and (3).

$$
\begin{aligned}
& \mathbf{F}=\left(\begin{array}{cc}
1 & \Delta t \\
0 & 1
\end{array}\right) \otimes \mathbf{I}_{2} \\
& \mathbf{Q}_{k}=q_{0} \mathbf{I}_{2} \otimes\left(\begin{array}{cc}
\frac{1}{3} \Delta t^{3} & \frac{1}{2} \Delta t^{2} \\
\frac{1}{2} \Delta t^{2} & \Delta t
\end{array}\right)
\end{aligned}
$$

where $\otimes$ is a symbol of kronecker product. $\Delta t$ denotes the sample interval. $q_{0}$ denotes the density of process noise. $I_{2}$ is a $2 \times 2$ unit matrix.

\subsection{Observation model}

Define all the received signals $\mathbf{r}=\left[\mathbf{r}_{1}, \ldots, \mathbf{r}_{n}, \ldots, \mathbf{r}_{N}\right]^{\mathrm{T}}$, where $\mathbf{r}_{n}$ is the signals received from the $n$th receiver. It can be expressed as

$$
\mathbf{r}_{n}(t)=\sum_{q=1}^{Q} \sum_{m=1}^{M} \sqrt{\alpha_{m q n} p_{m}} \xi_{m q n} s_{m}\left(t-\tau_{m q n}\right) e^{-j \omega_{m q n} t}+\mathbf{w}_{n}(t)
$$

where $m q n$ denotes the signal propagation path. The signal transmitted from the $m$ th transmitter is reflected by the $q$ th target, and the reflected signal is received by the $n$th receiver. $\alpha_{m q n}$ represents the path loss on propagation path $m q n, \alpha_{m q n} \propto 1 /\left(R_{m}^{q} R_{n}^{q}\right)^{2}$, $R_{m}^{q}$, and $R_{n}^{q}$ respectively denote the range from the $m$ th transmitter to the $q$ th target and the range from the $n$th receiver to the $q$ th target, which are of the form

$$
\left\{\begin{array}{l}
R_{m}^{q}=\sqrt{\left(x_{m}^{t}-x^{q}\right)^{2}+\left(y_{m}^{t}-y^{q}\right)^{2}} \\
R_{n}^{q}=\sqrt{\left(x_{n}^{r}-x^{q}\right)^{2}+\left(y_{n}^{r}-y^{q}\right)^{2}}
\end{array}\right.
$$

$\xi_{m q n}$ is the deterministic and complex target reflection coefficient. Let $\tau_{m q n}$ denote the signal propagation time on path $m q n$, satisfying $\tau_{m q n}=\left(R_{m}^{q}+R_{n}^{q}\right) / \mathrm{c}$, where $c$ is the light speed. $\omega_{m q n}$ is the Doppler frequency shift due to target moving, satisfying

$\omega_{m q n}=-\frac{2 \pi}{\lambda}\left[\left(\cos \phi_{m}^{q}+\cos \phi_{n}^{q}\right) v_{x}^{q}+\left(\sin \phi_{m}^{q}+\sin \phi_{n}^{q}\right) v_{y}^{q}\right]$

The term $\lambda$ is signal wavelength. $\phi_{m}^{q}$ and $\phi_{n}^{q}$ respectively represent the observation angle from the $m$ th transmitter and the $n$th receiver to the qth target. The term $\mathbf{w}_{n}(t)$ represents zero-mean and complex white Gaussian noise with autocorrelation function $\sigma_{w}^{2}$ $\delta(\tau)$.

At state $k$, the fusion center collects the range and Doppler information to estimate target states. The observation model for the $q$ th target can be expressed as

$$
\mathbf{z}_{k}^{q}=f\left(\mathbf{x}_{k}^{q}\right)+\mathbf{n}_{k}^{q}
$$

where $\mathbf{z}_{k}^{q}$ is the observed vector, including signal propagation time and Doppler frequency shift. $\mathbf{z}_{k}^{q}=\left[\left(\mathbf{\tau}_{k}^{q}\right)^{\mathrm{T}}\left(\boldsymbol{\omega}_{k}^{q}\right)^{\mathrm{T}}\right]^{\mathrm{T}}$,

$$
\left\{\begin{array}{l}
\mathbf{\tau}_{k}^{q}=\left[\tau_{1 q 1, k}, \ldots, \tau_{1 q n, k}, \ldots, \tau_{M q 1, k}, \ldots \tau_{M q N, k}\right]^{\mathrm{T}} \\
\boldsymbol{\omega}_{k}^{q}=\left[\omega_{1 q 1, k}, \ldots, \omega_{1 q n, k}, \ldots, \omega_{M q 1, k}, \ldots, \omega_{M q N, k}\right]^{\mathrm{T}}
\end{array}\right.
$$

$f\left(\right.$.) denotes the nonlinear observation process. $\mathbf{n}_{k}^{q}$ is observation noise.

Extend Kalman filter (EKF) is employed for targets tracking in this paper.

\subsection{Performance metric}

At high signal to noise ratio (SNR), BCRB can provide lower bound of parameter estimation error for target tracking [25]. According to [17], Bayesian information matrix (BIM) for the $q$ th target tracking is 


$$
\mathbf{J}_{\mathrm{B}}\left(\mathbf{x}_{k}^{q}\right)=\left[\mathbf{Q}_{k-1}+\mathbf{F J}_{\mathrm{B}}{ }^{-1}\left(\mathbf{x}_{k-1}^{q}\right) \mathbf{F}^{\mathrm{T}}\right]^{-1}+\mathbf{J}_{\mathrm{D}}\left(\mathbf{x}_{k}^{q}\right)
$$

where $\mathbf{F}$ is state transition matrix, $\mathbf{J}_{\mathrm{D}}\left(\mathbf{x}_{k}^{q}\right)$ is the fisher information matrix (FIM) of $\mathbf{x}_{k}^{q}$, which can be obtained by applying the chain rule [26],

$$
\mathbf{J}_{\mathrm{D}}\left(\mathbf{x}_{k}^{q}\right)=\mathrm{E}\left[\mathbf{Y}\left(\mathbf{x}_{k}^{q}\right) \mathbf{J}_{\mathrm{D}}\left(\mathbf{z}_{k}^{q}\right) \mathbf{Y}^{T}\left(\mathbf{x}_{k}^{q}\right)\right]
$$

where $\mathrm{E}[\cdot]$ denotes the expectation. $\mathbf{J}_{\mathrm{D}}\left(\mathbf{z}_{k}^{q}\right)$ is the FIM of $\mathbf{z}_{k}^{q}$, which can be calculated by the conditional probability density function,

$$
\ln \left[p\left(\mathbf{r} \mid \mathbf{z}_{k}^{q}\right)\right] \alpha_{-} \frac{1}{\sigma_{w}^{2}} \sum_{n=1}^{N} \int_{T}\left|\boldsymbol{r}_{n}^{q}(t)-\sum_{m=1}^{M} \sqrt{\alpha_{m q n} p_{m}} \xi_{m q n} s_{m}\left(t-\tau_{m q n}\right) e^{-j w_{m q n} t}\right|^{2} d t
$$

where $r_{n}^{q}(t)$ is the signals reflected by the $q$ th target and received from the $n$th receiver. Therefore, we can get

$$
\mathbf{J}_{\mathrm{D}}\left(\mathbf{z}_{k}^{q}\right)=8 \pi^{2} \operatorname{diag}\left(\left|\sqrt{\alpha_{m q n}} \xi_{m q n}\right|^{2} / \sigma_{w}^{2} \cdot f_{r} t_{m} p_{m}\right)
$$

where $f_{\mathrm{r}}$ is pulse repetition frequency. $\operatorname{diag}\{\cdot\}$ denotes matrix diagonalization. $\mathbf{Y}\left(\mathbf{x}_{k}^{q}\right)$ is a Jacobi matrix, $\mathbf{Y}\left(\mathbf{x}_{k}^{q}\right)=\Delta_{\mathbf{x}_{k}^{q}} z_{k}^{q}$, the derivation of $\mathbf{z}_{k}^{q}$ with respect to $\mathbf{x}_{k}^{q}$. $\mathbf{Y}\left(\mathbf{x}_{k}^{q}\right)$ is calculated by

$$
\begin{aligned}
\mathbf{Y}\left(\mathbf{x}_{k}^{q}\right) & =\Delta_{\mathbf{x}_{k}^{q} \mathbf{z}_{k}^{q}} \\
& =\left[\begin{array}{llllll}
\Delta_{x_{k}^{q}} \boldsymbol{\tau}_{k}^{q} & \Delta_{y_{k}^{q}} \boldsymbol{\tau}_{k}^{q} & \Delta_{v_{x, k}^{q}} & \boldsymbol{\tau}_{k}^{q} & \Delta_{v_{y, k}^{q}} \boldsymbol{\tau}_{k}^{q} \\
\Delta_{x_{k}^{q}}^{q} \boldsymbol{\omega}_{k}^{q} & \Delta_{y_{k}^{q}}^{q} \boldsymbol{\omega}_{k}^{q} & \Delta_{v_{x, k}^{q}} \boldsymbol{\omega}_{k}^{q} & \Delta_{v_{y, k}^{q}} \boldsymbol{\omega}_{k}^{q}
\end{array}\right]^{\mathrm{T}}
\end{aligned}
$$

where $\Delta_{x_{k}^{q}} \boldsymbol{\tau}_{k}^{q}, \Delta_{y_{k}^{q}} \boldsymbol{\tau}_{k}^{q}, \Delta_{v_{x, k}^{q}} \boldsymbol{\tau}_{k}^{q}, \Delta_{v_{y, k}^{q}} \boldsymbol{\tau}_{k}^{q}, \Delta_{x_{k}^{q}} \boldsymbol{\omega}_{k}^{q}, \Delta_{y_{k}^{q}} \boldsymbol{\omega}_{k}^{q}, \Delta_{v_{x, k}^{q}} \boldsymbol{\omega}_{k}^{q}$, $\Delta_{v_{\gamma, k}^{q}} \boldsymbol{\omega}_{k}^{q}$ can be easily calculated by targets state and antenna layout, which can be expressed as (14) and (15).

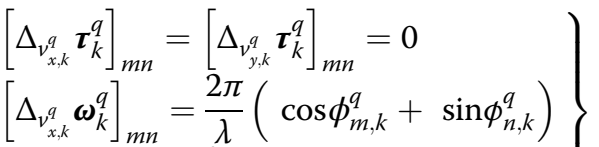

$$
\begin{aligned}
& \left.\left[\Delta_{v_{y, k}^{q}} \boldsymbol{\omega}_{k}^{q}\right]_{m n}=\frac{2 \pi}{\lambda}\left(\sin \phi_{m, k}^{q}+\cos \phi_{n, k}^{q}\right)\right)
\end{aligned}
$$

The first item of $\mathbf{J}_{\mathrm{B}}\left(\mathbf{x}_{k}^{q}\right)$ in (9) is just connected with the BIM and motion model at state $k-1$, which can be regarded as constant at state $k$. Since the BIM mainly depends on the second item $\mathbf{J}_{D}$, we propose an approximate method for the theoretical analysis with $\mathbf{J}_{B} \approx \mathbf{J}_{D}$ where $\mathbf{J}_{D}$ embraces the major information about resource function when the system resource is sufficient. Therefore, the theoretical analysis in this paper is an approximate method. Define $\mathbf{C}_{q}$ as the Bayesian Cramer-Rao matrix for the $q$ th target by inverting the FIM, $\mathbf{C}_{q}=\left[\mathbf{J}_{\mathrm{B}}\left(\mathbf{x}_{k}^{q}\right)\right]^{-1}$, whose diagonal elements are the lower-bounds on the variance of the target states estimation. For the qth target, with respect to the original velocity variables $v_{x}^{q}, v_{y}^{q}$, the estimated value $\hat{v}_{x}^{q}, \hat{v}_{y}^{q}$, meets $\operatorname{var}\left(\hat{v}_{x}^{q}\right)+\operatorname{var}\left(\hat{v}_{y}^{q}\right) \geq \operatorname{diag}_{C_{q}}(3)+\operatorname{diag}_{C_{q}}(4)$, where $\operatorname{var}(\cdot)$ denotes variance operator and $\operatorname{diag}_{C_{q}}(3)$ and $\operatorname{diag}_{C_{q}}(4)$ respectively denotes the CRLB for $v_{x}^{q}$ and $v_{y}^{q}$.

According to [19], the BCRB of velocity estimation for the $q$ th target can be derived as

$$
\begin{aligned}
V^{q} & =\operatorname{diag}_{C_{q}}(3)+\operatorname{diag}_{C_{q}}(4) \\
& =\frac{g^{q}+h^{q}}{\eta \cdot(2 \pi / \lambda)^{2}\left[g^{q} h^{q}-\left(z^{q}\right)^{2}\right]}
\end{aligned}
$$

where $g^{q}, h^{q}$, and $z^{q}$ are defined as $\left\{\begin{array}{l}g^{q}=\sum_{n=1}^{N} \sum_{m=1}^{M}\left|\sqrt{\alpha_{m q n}} \xi_{m q n}\right|^{2}\left(\cos \phi_{m}^{q}+\cos \phi_{n}^{q}\right)^{2} p_{m} t_{m}^{2} f_{m}^{\mathrm{t}} f_{n}^{\mathrm{r}} \\ h^{q}=\sum_{n=1}^{N} \sum_{m=1}^{M}\left|\sqrt{\alpha_{m q n}} \xi_{m q n}\right|^{2}\left(\sin \phi_{m}^{q}+\sin \phi_{n}^{q}\right)^{2} p_{m} t_{m}^{2} f_{m}^{\mathrm{t}} f_{n}^{\mathrm{r}} \\ z^{q}=\sum_{n=1}^{N} \sum_{m=1}^{M}\left|\sqrt{\alpha_{m q n}} \xi_{m q n}\right|^{2}\left(\sin \phi_{m}^{q}+\sin \phi_{n}^{q}\right)\left(\cos \phi_{m}^{q}+\cos \phi_{n}^{q}\right) p_{m} t_{m}^{2} f_{m}^{\mathrm{t}} f_{n}^{\mathrm{r}}\end{array}\right.$

where $\eta=8 \pi^{2} / \sigma_{w}^{2}$. Introduce a vector of binary variables $f_{m}^{\mathrm{t}}, f_{n}^{\mathrm{r}} \in\{0,1\}$, respectively representing the selection variable for the $m$ th transmitter and the $n$th receiver,

$$
\left.\begin{array}{l}
{\left[\Delta_{x_{k}^{q}} \boldsymbol{\tau}_{k}^{q}\right]_{m n}=\frac{1}{\mathrm{c}}\left(\cos \phi_{m, k}^{q}+\sin \phi_{n, k}^{q}\right), \quad\left[\Delta_{y_{k}^{q}} \boldsymbol{\tau}_{k}^{q}\right]_{m n}=\frac{1}{\mathrm{c}}\left(\sin \phi_{m, k}^{q}+\cos \phi_{n, k}^{q}\right)} \\
{\left[\Delta_{x_{k}^{q}} \boldsymbol{\omega}_{k}^{q}\right]_{m n}=-\frac{2 \pi}{\lambda}\left\{-v_{x, k}^{q}\left(\frac{\sin ^{2} \phi_{m, k}^{q}}{R_{m, k}^{q}}+\frac{\sin ^{2} \phi_{n, k}^{q}}{R_{n, k}^{q}}\right)+v_{y, k}^{q}\left(\frac{\sin \phi_{m, k}^{q} \cos \phi_{m, k}^{q}}{R_{m, k}^{q}}+\frac{\sin \phi_{n, k}^{q} \cos \phi_{n, k}^{q}}{R_{n, k}^{q}}\right)\right\}} \\
{\left[\Delta_{y_{k}^{q}} \boldsymbol{\omega}_{k}^{q}\right]_{m n}=-\frac{2 \pi}{\lambda}\left\{v_{y, k}^{q}\left(\frac{\cos ^{2} \phi_{m, k}^{q}}{R_{m, k}^{q}}+\frac{\cos ^{2} \phi_{n, k}^{q}}{R_{n, k}^{q}}\right)-v_{x, k}^{q}\left(\frac{\sin \phi_{m, k}^{q} \cos \phi_{m, k}^{q}}{R_{m, k}^{q}}+\frac{\sin \phi_{n, k}^{q} \cos \phi_{n, k}^{q}}{R_{n, k}^{q}}\right)\right\}}
\end{array}\right\}
$$


where 0 denotes abandoned and 1 denotes selected. The selection vectors for transmitters and receivers can be expressed as $\mathbf{f}_{\mathrm{t}}=\left[f_{1}^{\mathrm{t}}, \ldots, f_{m}^{\mathrm{t}}, \ldots, f_{M}^{\mathrm{t}}\right]^{\mathrm{T}}$ and $\mathbf{f}_{\mathrm{r}}=\left[f_{1}^{\mathrm{r}}, \ldots, f_{n}^{\mathrm{r}}, \ldots, f_{N}^{\mathrm{r}}\right]^{\mathrm{T}}$.

According to [23], we can define $\zeta_{m q n}=4 \eta \cdot(2 \pi / \lambda)^{2}$ $\cos ^{2}\left(\frac{\phi_{m}^{q}-\phi_{n}^{q}}{2}\right)\left|\sqrt{\alpha_{m q n}} \xi_{m q n}\right|^{2}$, and the function of (16) can be transformed as

$$
V^{q}\left(\mathbf{f}_{\mathrm{t}}, \mathbf{f}_{\mathrm{r}}, \mathbf{p}, \mathbf{t}\right)=\frac{4 \cdot \boldsymbol{F}_{\mathrm{r}}^{\mathrm{T}} \mathbf{H}^{q} \mathbf{v}}{\mathbf{v}^{\mathrm{T}}\left(\mathbf{H}^{q}\right)^{\mathrm{T}} \boldsymbol{\Sigma}^{q} \mathbf{H}^{q} \mathbf{v}}
$$

where $\boldsymbol{F}_{\mathbf{r}}=\mathbf{f}_{\mathrm{r}} \otimes \mathbf{1}, \mathbf{v}=\mathbf{p} \odot \mathbf{t}^{2} \odot \mathbf{f}_{\mathbf{v}} \mathbf{H}^{q}=\left[\mathbf{H}_{1}^{q}, \ldots, \mathbf{H}_{n}^{q}, \ldots, \mathbf{H}_{N}^{q}\right]^{\mathrm{T}}$, $\mathbf{H}_{n}^{q}=\operatorname{diag}\left\{\zeta_{1 q n}, \ldots, \zeta_{m q n}, \ldots, \zeta_{M q n}\right\} . \Sigma^{q}$ is a symmetric matrix of $\operatorname{rank}\left(\Sigma^{q}\right) \leq 3$, given by

$$
\boldsymbol{\Sigma}^{q}=\boldsymbol{F}_{\mathrm{r}} \boldsymbol{F}_{\mathrm{r}}^{\mathrm{T}}-\left(\boldsymbol{F}_{\mathrm{r}} \odot \boldsymbol{C}^{q}\right)\left(\boldsymbol{F}_{\mathrm{r}} \odot \boldsymbol{C}^{q}\right)^{\mathrm{T}}-\left(\boldsymbol{F}_{\mathrm{r}} \odot \boldsymbol{S}^{q}\right)\left(\boldsymbol{F}_{\mathrm{r}} \odot \boldsymbol{S}^{q}\right)^{\mathrm{T}}
$$

where

$$
\begin{cases}\mathbf{C}^{q}=\left[\mathbf{C}_{1}^{q}, \ldots, \mathbf{C}_{m}^{q}, \ldots, \mathbf{C}_{M}^{q}\right]^{\mathrm{T}} & \mathbf{S}^{q}=\left[\mathbf{S}_{1}^{q}, \ldots, \mathbf{S}_{m}^{q}, \ldots, \mathbf{S}_{M}^{q}\right]^{\mathrm{T}} \\ \mathbf{C}_{n}^{q}=\left[C_{1 n}^{q}, \ldots, C_{m n}^{q}, \ldots, C_{M n}^{q}\right] & \mathbf{S}_{n}^{q}=\left[S_{1 n}^{q}, \ldots, S_{m n}^{q}, \ldots, S_{M n}^{q}\right] \\ C_{m n}^{q}=\cos \left(\phi_{m}^{q}+\phi_{n}^{q}\right) & S_{m n}^{q}=\sin \left(\phi_{m}^{q}+\phi_{n}^{q}\right)\end{cases}
$$

Therefore, (18) is the velocity estimation error low bound, which can be used as the performance metric for velocity estimation.

\section{Methods}

In this section, we formulate the resource allocation model and propose a three-step suboptimal method by transforming the problem into SOCP problems.

\subsection{Problem formulation}

Due to different tasks for targets, different velocity estimation requirements for each target are considered. We define the target set as $\left\{1, \ldots, q, \ldots, q^{*}, \ldots, Q\right\}$, including general targets $\{1, \ldots, q, \ldots, Q\}$ and one key target $q^{*}$. When the transmitters, receivers, and transmitted power and signal time are limited and relatively sufficient, restricted by given velocity estimation MSE requirements for general targets, the resource allocation model for better estimation performance of the key target $q^{\prime \prime}$ can be formulated as

$$
\begin{aligned}
& \min _{\mathbf{f}^{\mathrm{t}}, \mathbf{f}^{\mathrm{t}}, \mathbf{p}, \mathbf{t}} V^{q^{*}}\left(\mathbf{f}_{\mathrm{t}}, \mathbf{f}_{\mathrm{r}}, \mathbf{p}, \mathbf{t}\right) \\
& \text { s.t. }\left\{\begin{array}{l}
\mathbf{1}^{\mathrm{T}} \mathbf{p}=P_{\text {total }} \\
\mathbf{1}^{\mathrm{T}} \mathbf{t}=T_{\text {total }} \\
\mathbf{1}^{\mathrm{T}} \mathbf{f}_{\mathrm{t}} \leq \mathrm{Kt} \\
\mathbf{1}^{\mathrm{T}} \mathbf{f}_{\mathrm{r}} \leq \mathrm{Kr} \\
0 \leq p_{m} \leq p_{\max }, m \in\{1, \ldots, M\} \\
0 \leq t_{m} \leq t_{\max }, m \in\{1, \ldots, M\} \\
f_{m}^{\mathrm{t}}, f_{n}^{\mathrm{r}} \in\{0,1\}, m \in\{1, \ldots, M\}, n \in\{1, \ldots, N\} \\
V^{q}\left(\mathbf{f}_{\mathrm{t}}, \mathbf{f}_{\mathrm{r}}, \mathbf{p}, \mathbf{t}\right) \leq \mathrm{MSE}^{q}, q \in\{1, \ldots, Q\} / q^{*}
\end{array}\right.
\end{aligned}
$$

where $P_{\text {total }}$ and $T_{\text {total }}$ respectively denote total transmitted power and total signal time. The corresponding upper bounds are given by $p_{\max }$ and $t_{\max }$. Kt and $\mathrm{Kr}$ respectively denote the allowed maximal transmitter number and receiver number. $\mathrm{MSE}^{q}$ is the allowed velocity estimation MSE for the qth target.

\subsection{Three-step SOCP optimization method}

Since antenna variables are Boolean type and transmitted parameters are successive type, problem in (21) can be classified as a mixed Boolean optimization problem, which can be separated into some suboptimal problems and solved by a cyclic minimization method [27, 28]. Here, we introduce an auxiliary variable $\lambda^{q}$ for each target, $V^{q}\left(\mathbf{f}_{\mathfrak{t}}, \mathbf{f}_{\mathfrak{r}} \mathbf{p}, \mathbf{t}\right) \leq\left(\lambda^{q}\right)^{-1}$. Generally, the MSE requirements of general targets $\{1, \ldots, q, \ldots, Q\}$ are larger than that of key target $q^{*}$. After meeting the general targets' estimation MSE by maximizing resource utilization, all the remaining resources can be allocated to the key target for the best performance. The optimization model can be transformed as (22) where the objective function is about $\lambda^{q^{*}}$ to get its best tracking performance for the key target and the last two constraints are about general targets $\lambda^{q}, \quad q \in\{1, \ldots, Q\} / q^{*}$, with their given MSE requirements.

$$
\begin{aligned}
& \min _{\mathbf{f}^{\mathrm{t}}, \mathbf{f}^{\mathrm{t}} \mathbf{p}, \mathbf{t}}\left(\lambda^{q^{*}}\right)^{-1} \\
& \text { s.t. }\left\{\begin{array}{l}
\mathbf{1}^{\mathrm{T}} \mathbf{p}=P_{\text {total }} \\
\mathbf{1}^{\mathrm{T}} \mathbf{t}=T_{\text {total }} \\
\mathbf{1}^{\mathrm{T}} \mathbf{f}_{\mathrm{t}} \leq \mathrm{Kt} \\
\mathbf{1}^{\mathrm{T}} \mathbf{f}_{\mathrm{r}} \leq \mathrm{Kr} \\
0 \leq p_{m} \leq p_{\max }, m \in\{1, \ldots, M\} \\
0 \leq t_{m} \leq t_{\max }, m \in\{1, \ldots, M\} \\
f_{m}^{\mathrm{t}}, f_{n}^{\mathrm{r}} \in\{0,1\}, m \in\{1, \ldots, M\}, n \in\{1, \ldots, N\} \\
V^{q}\left(\mathbf{f}_{\mathrm{t}}, \mathbf{f}_{\mathrm{r}}, \mathbf{p}, \mathbf{t}\right) \leq\left(\lambda^{q}\right)^{-1}, q \in\{1, \ldots, Q\} / q^{*} \\
0 \leq\left(\lambda^{q}\right)^{-1} \leq \mathrm{MSE}^{q}, q \in\{1, \ldots, Q\} / q^{*}
\end{array}\right.
\end{aligned}
$$

where the objective function of (22) is nonlinear. Considering that $\min _{\mathbf{f}^{t}, \mathbf{f}^{\mathrm{r}}, \mathbf{p}, \mathbf{t}}\left(\lambda^{q^{*}}\right)^{-1}$ can be equivalent to $\min _{\mathbf{f}^{\mathrm{t}}, \mathbf{f}^{\mathbf{t}}, \mathbf{p}, \mathbf{t}}-\lambda^{q^{*}}$. For simplicity, (22) can be further transformed as 


$$
\begin{aligned}
& \min _{\mathbf{f}^{\mathbf{t}}, \mathbf{f}^{\mathrm{t}}, \mathbf{p}, \mathbf{t}}-\lambda^{q^{*}} \\
& \text { s.t. }\left\{\begin{array}{l}
\mathbf{1}^{\mathrm{T}} \mathbf{p}=P_{\text {total }} \\
\mathbf{1}^{\mathrm{T}} \mathbf{t}=T_{\text {total }} \\
\mathbf{1}^{\mathrm{T}} \mathbf{f}_{\mathrm{t}} \leq \mathrm{Kt} \\
\mathbf{1}^{\mathrm{T}} \mathbf{f}_{\mathrm{r}} \leq \mathrm{Kr} \\
0 \leq p_{m} \leq p_{\max }, m \in\{1, \ldots, M\} \\
0 \leq t_{m} \leq t_{\max }, m \in\{1, \ldots, M\} \\
f_{m}^{\mathrm{t}}, f_{n}^{\mathrm{r}} \in\{0,1\}, m \in\{1, \ldots, M\}, n \in\{1, \ldots, N\} \\
\lambda^{q} \times V^{q}\left(\mathbf{f}_{\mathrm{t}}, \mathbf{f}_{\mathrm{r}}, \mathbf{p}, \mathbf{t}\right) \leq 1, q \in\{1, \ldots, Q\} / q^{*} \\
\lambda^{q} \geq 1 / \mathrm{MSE}^{q}, q \in\{1, \ldots, Q\} / q^{*}
\end{array}\right.
\end{aligned}
$$

To simplify the problem model, proposition 1 will be applied to (23).

Proposition 1: The optimal transmitted power $\mathbf{p}^{\text {opt }}$ and time $\mathbf{t}^{\text {opt }}$ of problem (23) are related through

$$
\mathbf{p}^{\text {opt }}=\frac{P_{\text {total }}}{T_{\text {Total }}} \mathbf{t}^{\text {opt }}
$$

\section{Proof: See Appendix A.}

As this proposition, (23) can be separated into a problem of three optimal variables. According to [23], the antenna selection variables can be relaxed to successive variables. The objective function of (23) is linear, while the eighth constraint is convex function of $\mathbf{v}$ and $\boldsymbol{F}_{\mathrm{r}}$. Besides, $\Sigma^{q}$ is a matrix with a low rank. The convexity and low rank properties can be exploited to develop efficient allocation method. Consequently, the joint allocation formulation is equivalent to a SOCP problem. To transform the optimization model to a SOCP form, proposition 2 will be applied to model (23).

Proposition 2: The eighth constraint of (23) $\lambda^{q} \times V^{q}\left(\mathbf{f}_{\mathrm{t}}\right.$, $\left.\mathbf{f}_{\mathbf{p}} \mathbf{p}, \mathbf{t}\right) \leq 1$ is equivalent to the SOCP form

$$
\left\|\left(\boldsymbol{F}_{\mathrm{r}} \odot \mathbf{A}^{q}\right) \mathbf{y}+\mathbf{b}\right\| \leq\left(\boldsymbol{F}_{\mathrm{r}}\right)^{\mathrm{T}} \mathbf{y}-2 \lambda^{q}
$$

where $\mathbf{A}^{q}=\left[\mathbf{C}^{q}, \mathbf{S}^{q}, \mathbf{0}\right]^{\mathrm{T}}, \mathbf{y}=\mathbf{H}^{q} \mathbf{v}, \mathbf{b}=\left[0,0,2 \lambda^{q}\right]^{\mathrm{T}}$.

Proof: See Appendix B.

Here, the transmitters, receivers, transmitted power, and signal time are optimized in turn. The specific optimization process is presented as 1)-5).

1) Variables initialization

Relax the Boolean variables $f_{m}^{\mathrm{t}}, f_{n}^{\mathrm{r}} \in\{0,1\}$ to successive variables $f_{m}^{\mathrm{t}}, f_{n}^{\mathrm{r}} \in[0,1]$. Uniform allocation is employed for initial power vector $\mathbf{p}^{*}=\mathrm{P}_{\text {total }} / M \cdot \mathbf{1}$, initial time vector $\mathbf{t}^{*}=\mathrm{T}_{\text {total }} / M \cdot \mathbf{1}$. And all receivers are selected $\mathbf{f}_{\mathrm{r}}^{*}=\mathbf{1}$, $\mathbf{F}_{\mathrm{r}}^{*}=\mathbf{f}_{\mathrm{r}}^{*} \otimes \mathbf{1}$.

2) Transmitter selection

The transmitter selection formulation is equivalent to a SOCP as follows

$$
\begin{aligned}
& \min _{\mathbf{f}_{\mathrm{t}}}-\lambda_{1}^{q^{*}} \\
& \text { s.t. }\left\{\begin{array}{l}
\mathbf{1}^{\mathrm{T}} \mathbf{f}_{\mathrm{f}} \leq \mathrm{Kt} \\
0 \leq f_{m}^{t} \leq 1, m \in\{1, \ldots, M\} \\
\left\|\left(\boldsymbol{F}_{\mathrm{r}}^{*} \odot \mathbf{A}^{q}\right) \mathbf{H}^{q}\left(\mathbf{p}^{*} \odot\left(\boldsymbol{t}^{*}\right)^{2} \odot \mathbf{f}_{\mathbf{f}}\right)+\mathbf{b}_{1}\right\| \leq\left(\boldsymbol{F}_{\mathrm{r}}^{*}\right)^{\mathrm{T}} \mathbf{H}^{q}\left(\mathbf{p}^{*} \odot\left(\boldsymbol{t}^{*}\right)^{2} \odot \mathbf{f}_{\mathrm{t}}\right)-2 \lambda_{1}^{q} \\
\lambda_{1}^{q} \geq 1 / \mathrm{MSE}^{q}, q \in\{1, \ldots, Q\} / q^{*}
\end{array}\right.
\end{aligned}
$$

where $\mathbf{A}^{q}=\left[\mathbf{C}^{q}, \mathbf{S}^{q}, \mathbf{0}\right]^{\mathrm{T}}$ and $\mathbf{b}_{1}=\left[0,0,2 \lambda_{1}^{q}\right]^{\mathrm{T}}$. Different from (23), the object function and constraints for $\mathbf{f}_{\mathrm{t}}$ and $\lambda_{1}^{q}$ are linear in (26). The problem can be solved by a convex optimization method. In this case, we can get the solution $\mathbf{f}_{\mathrm{t}}^{*}$ for the relaxed problem, where $\mathbf{f}_{\mathrm{t}}^{*}$ can be fractional.

3) Receiver selection

Using the selection result $\mathbf{f}_{t}^{*}$, the receiver selection formulation can be transformed into a SOCP in a similar way

$$
\begin{array}{ll}
\min _{\mathbf{f}_{\mathrm{r}}}-\lambda_{2}^{q^{*}} \\
\text { s.t. }\left\{\begin{array}{l}
\mathbf{1}^{\mathrm{T}} \mathbf{f}_{\mathrm{r}} \leq \mathrm{Kr} \\
0 \leq f_{n}^{r} \leq 1, n \in\{1, \ldots, N\} \\
\left\|\left(\mathbf{F}_{\mathrm{r}} \odot \mathbf{A}^{q}\right) \mathbf{H}^{q} \mathbf{v}^{*}+\mathbf{b}_{2}\right\| \leq \mathbf{F}_{\mathrm{r}}{ }^{\mathrm{T}} \mathbf{H}^{q} \mathbf{v}^{*}-2 \lambda_{2}^{q} \\
\lambda_{2}^{q} \geq 1 / \mathrm{MSE}^{q}, q \in\{1, \ldots, Q\} / q^{*}
\end{array}\right.
\end{array}
$$

where $\mathbf{F}_{\mathrm{r}}=\mathbf{f}_{\mathrm{r}} \otimes \mathbf{1}$ and $\mathbf{b}_{2}=\left[0,0,2 \lambda_{2}^{q}\right]^{\mathrm{T}}$. Similarly, the solution $\mathbf{f}_{\mathrm{r}}^{*}$ and $\mathbf{F}_{\mathrm{r}}^{*}$ for relaxed problem (27) can be fractional.

4) Power and time allocation

After the antenna selection $\mathbf{f}_{\mathrm{t}}^{*}, \mathbf{f}_{\mathrm{r}}^{*}$, using Proposition 1 , the problem of joint transmitted power and time allocation can be expressed as (28) where $\mathbf{b}_{3}=\left[0,0,2 \lambda_{3}^{q}\right]^{\mathrm{T}}$. The optimal power and time allocation results are respectively $\mathbf{p}^{\prime \prime}$ and $\mathbf{t}^{*}$.

$$
\begin{aligned}
& \min _{\mathbf{p}}-\lambda_{3}^{q^{*}} \\
& \text { s.t. }\left\{\begin{array}{l}
\mathbf{1}^{\mathrm{T}} \mathbf{p}=P_{\text {total }} \\
\mathbf{1}^{\mathrm{T}} \mathbf{t}=T_{\text {total }} \\
\mathbf{t}=\frac{T_{\text {total }}}{P_{\text {total }}} \mathbf{p} \\
0 \leq p_{m} \leq p_{\max }, m \in\{1, \ldots, M\} \\
0 \leq t_{m} \leq t_{\max }, m \in\{1, \ldots, M\} \\
\left\|\left(\boldsymbol{F}_{\mathrm{r}}^{*} \odot \mathbf{A}^{q}\right) \mathbf{H}^{q}\left(\mathbf{p} \odot \mathbf{t}^{*} \odot \mathbf{f}_{\mathrm{t}}^{*}\right)+\mathbf{b}_{3}\right\| \leq\left(\boldsymbol{F}_{\mathrm{r}}^{*}\right)^{\mathrm{T}} \mathbf{H}^{q}\left(\mathbf{p} \odot \mathbf{t}^{2} \odot \mathbf{f}_{\mathrm{t}}^{*}\right)-2 \lambda_{3}^{q} \\
\lambda_{3}^{q} \geq 1 / \mathrm{MSE}^{q}, q \in\{1, \ldots, Q\} / q^{*}
\end{array}\right.
\end{aligned}
$$

\section{5) Result correction}

Jump to 2), circulation stops until the convergence is ensured for $-\lambda_{3}^{q^{*}}$. Then we will choose the Kt largest elements of $\mathbf{f}_{\mathrm{t}}^{*}$. Let $\mathbf{f}_{\mathrm{t}}^{\text {opt }}$ be the corresponding $0-1$ vector. Similarly, the Kr largest elements of $\mathbf{f}_{\mathrm{r}}^{*}$ are transformed into $\mathbf{f}_{\mathrm{r}}^{\text {opt }}$. Repeat 4) for one time, using $\mathbf{f}_{\mathrm{t}}^{\text {opt }}$ and $\mathbf{f}_{\mathrm{r}}^{\text {opt }}$. The optimal power $\mathbf{p}^{\text {opt }}$ and time $\mathbf{t}^{\text {opt }}$ can be efficiently obtained. Finally, correct $\mathbf{f}_{\mathrm{t}}^{\text {opt }}$ with $\mathbf{p}^{\text {opt }}$ and $\mathbf{t}^{\text {opt }}$. 


\section{Results and discussion}

Consider a distributed MIMO radar system with $M=6$, $N=6$. The allowed transmitter and receiver subsets are set to be $\mathrm{Kt}=4, \mathrm{Kr}=4$. The total transmitted power is $P_{\text {total }}=6 \mathrm{kw}$, the power upper bound for each transmitter is $p_{\max }=4 \mathrm{kw}$. And the corresponding time constraints are $T_{\text {total }}=0.6 \mathrm{~s}, t_{\max }=0.4 \mathrm{~s}$. In a $20 \mathrm{~km}$ $\times 20 \mathrm{~km}$ area, three targets are considered. In this paper, we define target 1 as the key target for better estimation performance. Target 2 and target 3 are general targets with larger MSE requirements. We assume that the system resources are enough for three targets. Under the premise of meeting general targets' requirements, the remaining resources can still achieve good performance for the key target. The velocity estimation MSE for three targets is $\mathrm{MSE}=\left[\mathrm{MSE}^{1}, \mathrm{MSE}^{2}, \mathrm{MSE}^{3}\right]$. In this paper, we discuss three different MSE requirements to test the proposed method. The detailed values and meanings for MSE are described in Table 1. We consider four different allocation methods and each method is described in Table 2 .

In this section, we compare the allocation results of different allocation methods and velocity estimation MSE requirements. To demonstrate the influence of antenna geometrical deployment on estimation performance, fixed distribution scenario and random distribution scenario for antennas and targets trajectory are respectively studied in Section 4.1 and 4.2. To clearly analyze and compare the allocation performance, we extract and study target states at one state.

\subsection{Fixed distribution scenario}

At one state, we obtain the geometrical deployment of antennas and targets location as Fig. 1. Three different MSE requirements in Table 1 are analyzed. In this section, different allocation methods are studied to improve target velocity estimation performance.

To evaluate the estimation performance for the key target, we assume the velocity estimation requirement is $\mathrm{MSE}=[$ inf, inf, $\mathrm{inf}] \mathrm{m}^{2} / \mathrm{s}^{2}$, which means only the key target is focused. The velocity estimation accuracy and

Table 1 The detailed value and meaning of different MSE

\begin{tabular}{|c|c|}
\hline $\begin{array}{l}\text { Discussed MSE } \\
\text { values }\left(\mathrm{m}^{2} / \mathrm{s}^{2}\right)\end{array}$ & Meaning of each MSE value \\
\hline $\mathrm{MSE}=[\mathrm{inf}, \mathrm{inf}, \mathrm{inf}]$ & $\begin{array}{l}\text { Ignore the target } 2 \text { and target } 3 \text {, all the resources } \\
\text { will be used for the key target. }\end{array}$ \\
\hline $\mathrm{MSE}=\left[\mathrm{inf}, 10^{2}, \mathrm{inf}\right]$ & $\begin{array}{l}\text { Ignore the target } 3 \text {. Two targets are considered. } \\
\text { The system resources will firstly meet target 2's } \\
\text { requirement, and all the remaining resources will } \\
\text { be used for key target. }\end{array}$ \\
\hline MSE $=\left[\right.$ inf $\left., 20^{2}, 20^{2}\right]$ & $\begin{array}{l}\text { Three targets are considered. The system } \\
\text { resources will firstly meet target } 2 \text { and target } 3 \text {, } \\
\text { and all the remaining resources will be used for } \\
\text { key target. }\end{array}$ \\
\hline
\end{tabular}

Table 2 Resource allocation methods

\begin{tabular}{|c|c|}
\hline $\begin{array}{l}\text { Allocation } \\
\text { methods }\end{array}$ & Meaning of method symbols \\
\hline$f_{t}, f_{r}$ & $\begin{array}{l}f_{t} \text { denotes transmitter vector. } f_{r} \text { denotes receiver vector. } \\
f_{t}, f_{r} \text { is a joint transmitter and receiver selection method } \\
\text { where transmitted power and signal time employ } \\
\text { uniform allocation. }\end{array}$ \\
\hline$f_{t}, f_{r}, p$ & $\begin{array}{l}p \text { denotes transmitted power vector. } f_{t}, f_{r,} p \text { is a joint } \\
\text { allocation method of antenna selection and } \\
\text { transmitted power allocation where signal time } \\
\text { employs uniform allocation. }\end{array}$ \\
\hline$f_{t}, f_{r}, t$ & $\begin{array}{l}t \text { denotes signal time vector } f_{t}, f_{r}, t \text { is a joint allocation } \\
\text { method of antenna selection and signal time allocation } \\
\text { where transmitted power employs uniform allocation. }\end{array}$ \\
\hline $\begin{array}{l}f_{t}, f_{r}, t, p \\
\text { (proposed) }\end{array}$ & $\begin{array}{l}f_{t}, f_{r}, t, p \text { is a proposed joint allocation method of } \\
\text { antenna selection and transmitted power, signal time } \\
\text { allocation. }\end{array}$ \\
\hline
\end{tabular}

resource allocation results are given in Fig. 2. The velocity estimation results show that more resource types mean larger controllable adjustable scope, and signal time has a more significant impact than transmitted power on estimation performance. The resource allocation results for transmitted power and time are respectively shown in Fig. 2b, c where more resources can be allocated to transmitter 2 and transmitter 5 close to the key target, which indicate that transmitters close to targets play an important part in target tracking.

Considering that velocity estimation MSE should be suited to the specific target task. Under velocity estimation requirements $\mathrm{MSE}=\left[\mathrm{inf}, 10^{2}\right.$, inf $] \mathrm{m}^{2} / \mathrm{s}^{2}$ and $\mathrm{MSE}=\left[\mathrm{inf}, 20^{2}, 20^{2}\right] \mathrm{m}^{2} / \mathrm{s}^{2}$, the allocation results are respectively shown in Figs. 3 and 4. For the former, the key target and target 2 are tracked together. In Fig. 3a, the last two allocation methods can meet given MSE requirements and achieve higher estimation accuracy. In Fig. 3b, c,

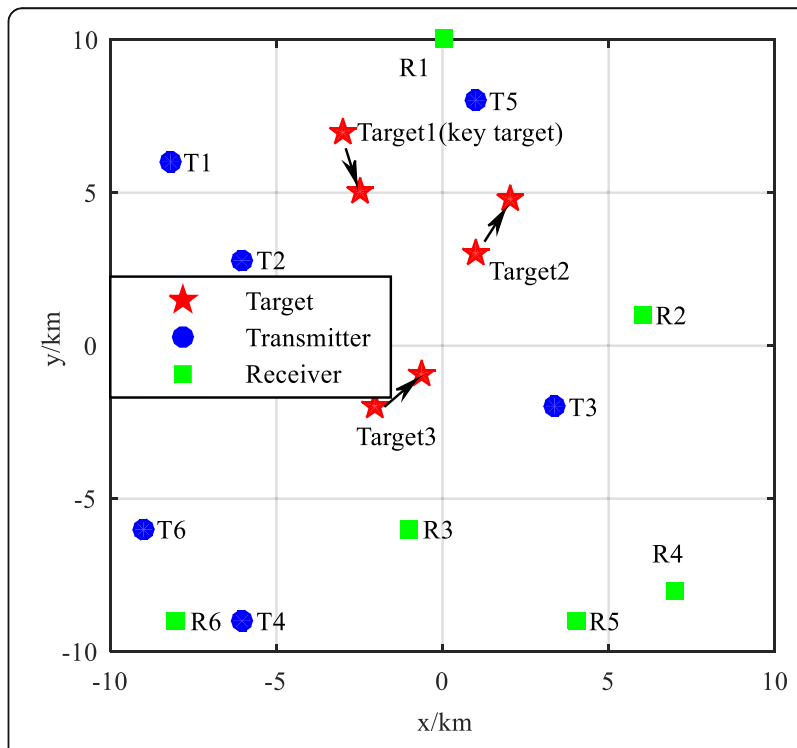

Fig. 1 Radars and targets layout 

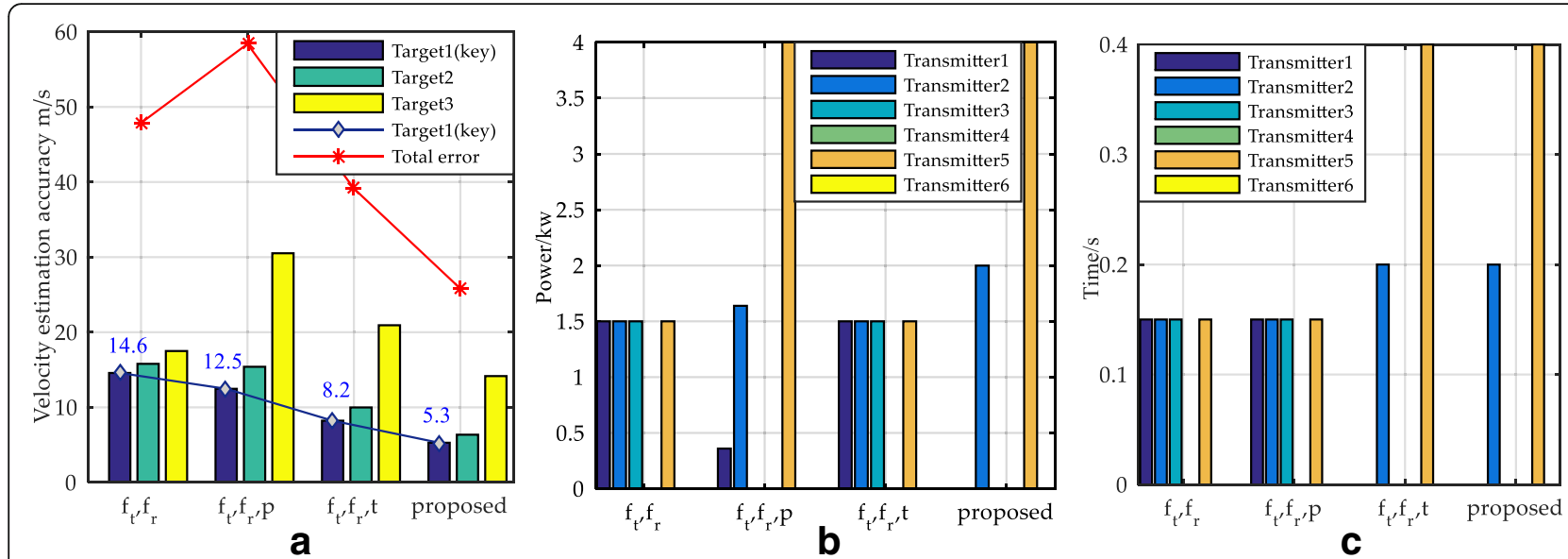

Fig. 2 Experimental results with MSE $=[\mathrm{inf}, \mathrm{inf}, \mathrm{inf}] \mathrm{m}^{2} / \mathrm{s}^{2}$. a Velocity estimation accuracy. b Power allocation results. c Time allocation results

transmitter 3 and transmitter 5 can get all the power and signal time, which is similar with that of Fig. 2. To get the best estimation performance for the key target, transmitter 5 close to the key target is allocated the major resources. For the later, three targets are focused. When meeting the general targets' demands, the key target can get the best performance. Moreover, transmitter 2 and transmitter 3 are both embraced for two general targets.

We compare the estimation performance of Figs. 2, 3, and 4 . With the increasing focused target number, the performance for key target is changing, which depends on the specific MSE requirements. Compared with the first three methods, the proposed joint method can get the best estimation performance for the key target and minimize the total MSE error.

\subsection{Random distribution scenario}

To better analyze the influence of system layout, random distribution scenario is simulated in this case, where targets, transmitters, and receivers are randomly distributed and the minimal distance among them is $2 \mathrm{~km}$. The performance is evaluated from the average value of 500 simulations.

Considering that the receivers always select the maximal receiver number, in a random distribution scenario, the velocity estimation accuracy and transmitter number with different resource allocation methods are shown in Figs. 5, 6, and 7 for the corresponding velocity estimation MSE. In Fig. 5a, since there is no estimation requirements for target 2 and target 3 , random experiments indicate that they get the approximate same estimation accuracy while the key target can get the best estimation performance. In Fig. 6a, using the first three methods, key target and target 3 can get the same estimation accuracy. Meanwhile, the estimation accuracy of target 2 is worse than its MSE requirement, which means that system resources are all focused on target 2 , inconsiderate to the key target when the general target's MSE requirement is hard to achieve. For the last proposed method, target 2 can achieve its MSE requirement

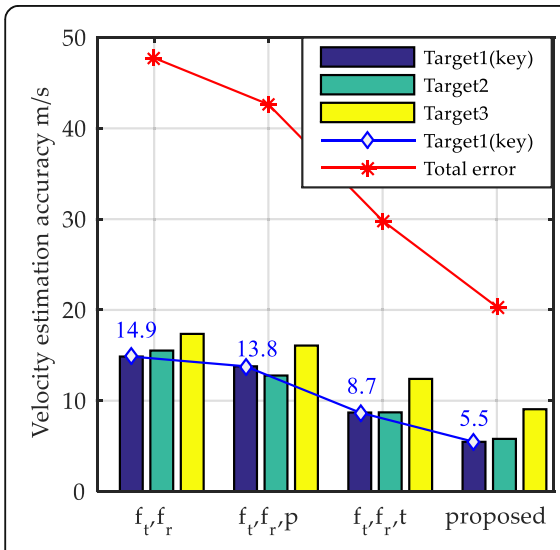

a

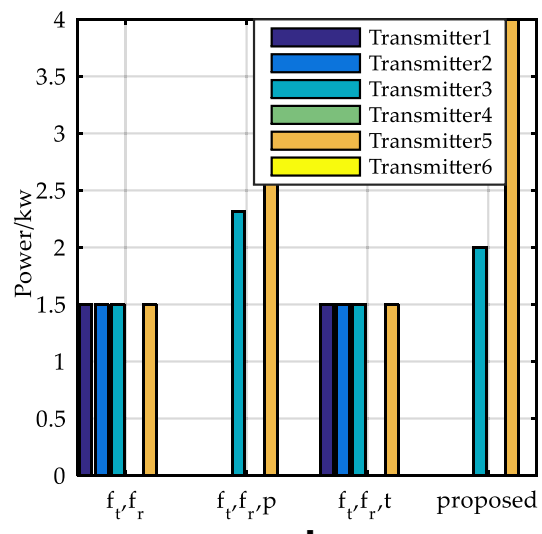

b

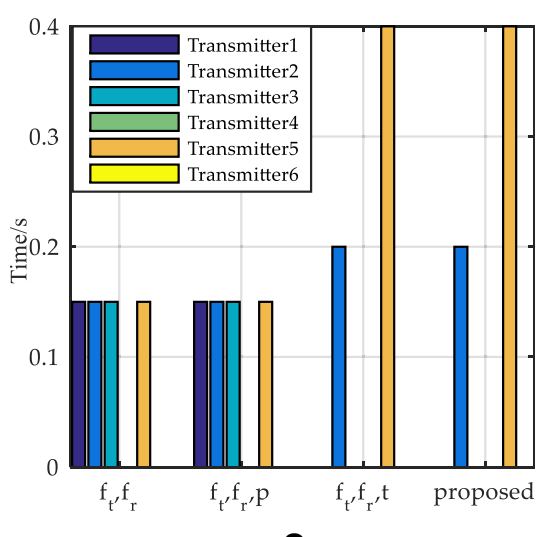

C

Fig. 3 Experimental results with $M S E=\left[\right.$ inf, $10^{2}$, inf $] \mathrm{m}^{2} / \mathrm{s}^{2}$. a Velocity estimation accuracy. b Power allocation results. c Time allocation results 


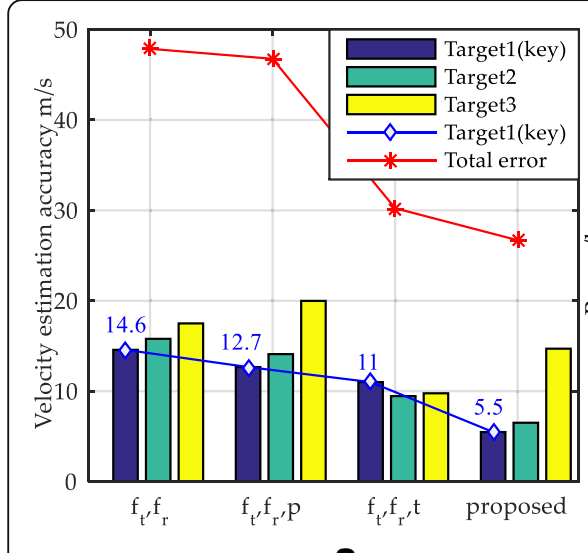

a

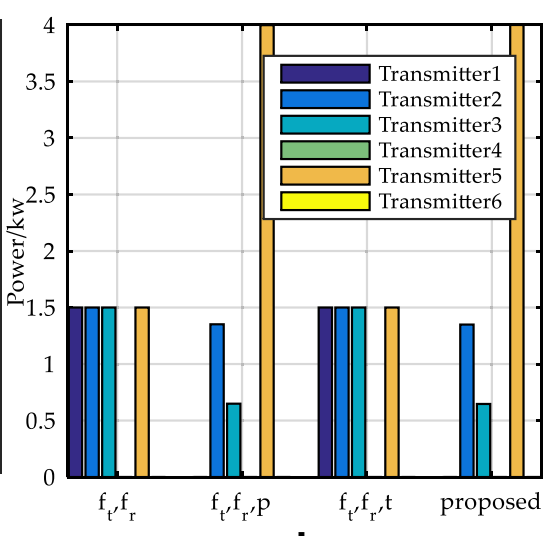

b

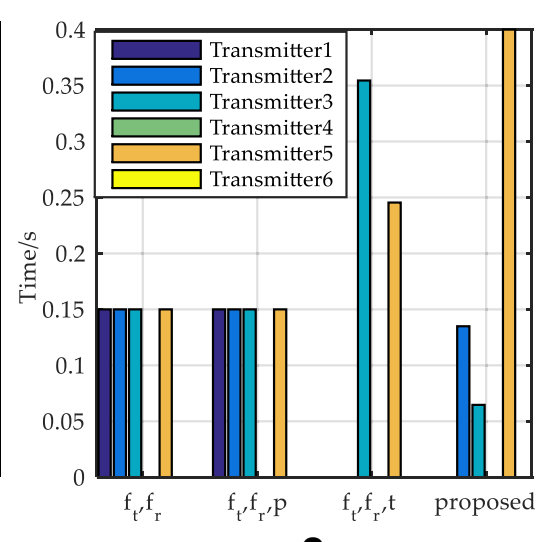

C

Fig. 4 Experimental results with $M S E=\left[i n f, 20^{2}, 20^{2}\right] \mathrm{m}^{2} / \mathrm{s}^{2}$. a Velocity estimation accuracy. b Power allocation results. c Time allocation results

and the key target get better estimation accuracy, which indicates that proposed joint allocation method can mostly improve the resource utilization and estimation performance. In Fig. 7a, though all methods can meet the MSE requirements, the proposed joint allocation method can further improve the estimation accuracy. Comparing the total estimation error of different allocation methods in Figs. 5a, 6a, and 7a, we can find the proposed method can get the minimal total velocity estimation error. The selected transmitter proportions for different MSE requirements and allocation methods are compared in Figs. 5b, 6b, and 7b where the results indicate that joint antenna and transmitted parameter allocation can reduce the selected transmitter number, in particular the proposed method selects the fewest transmitters. Moreover, the transmitter number is gradually increased with more target number focused.

In this case, for the defined system task, the joint allocation methods perform better than single antenna selection.
Moreover, the proposed method can reach to the minimal error using the fewest transmitters when meeting the velocity estimation MSE requirements. The results are consistent with that in the fixed distribution scenario.

\section{Conclusions}

In the distributed MIMO radar systems, a joint resource allocation method with transmitters, receivers, transmitted power, and signal time is proposed for better velocity estimation in multiple targets tracking system. In this work, we improve the estimation performance for key target when maximizing the system resource utilization of general targets. We formulate the optimization problem to a three-step suboptimal method where each optimization problem is transformed into a SOCP form by convex relaxation. Finally, an approximate optimal solution can be obtained.

In fixed distribution scenario, the simulations show that the proposed joint allocation method can meet the MSE
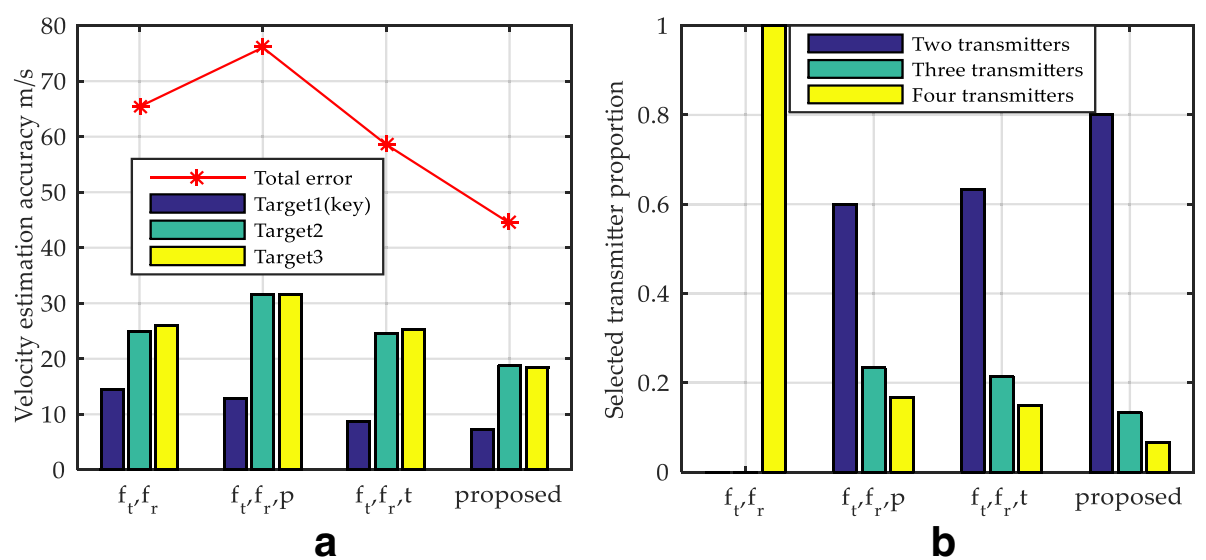

Fig. 5 Velocity estimation accuracy and transmitter number at random layout MSE $=[$ inf, inf, inf $] \mathrm{m}^{2} / \mathrm{s}^{2}$.a Velocity estimation accuracy $\mathbf{b}$ transmitter proportion 

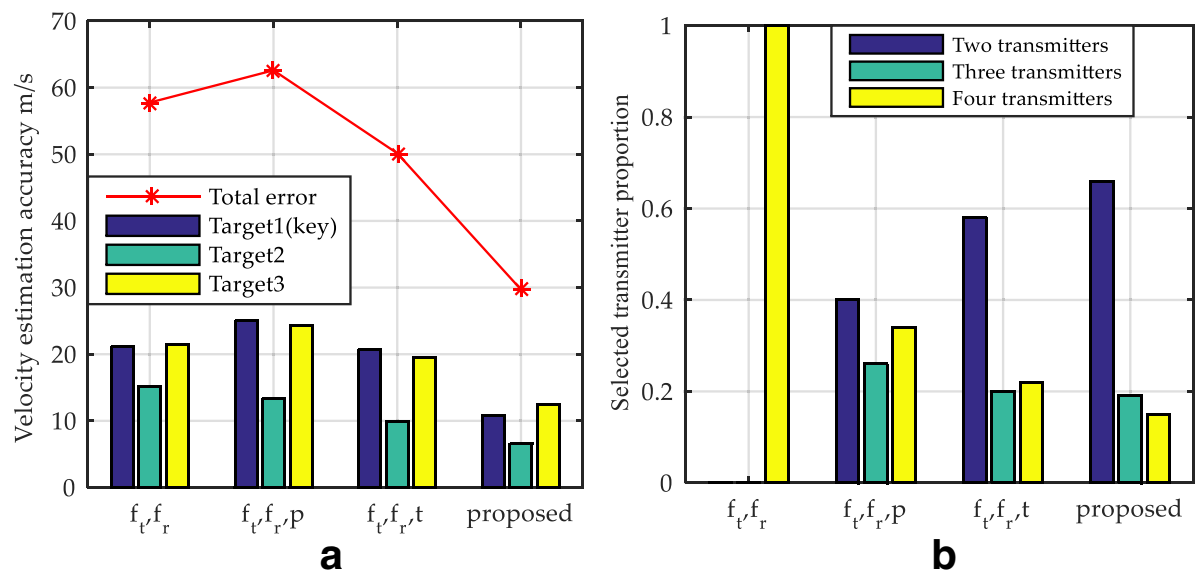

Fig. 6 Velocity estimation accuracy and transmitter number at random layout MSE $=\left[\mathrm{inf}, 10^{2}, \mathrm{inf}\right] \mathrm{m}^{2} / \mathrm{s}^{2}$. a Velocity estimation accuracy $\mathbf{b}$ Transmitter proportion

requirements with fewer transmitters and achieve the smaller total estimation error than other methods. By adjusting the estimation MSE requirements, the proposed method can control target number more flexibly when ensuring the demand of the key target. Compared with transmitted power, the signal time has more impact on the estimation performance. In addition, the random distribution scenario further validates the superiority of the proposed allocation method, which indicates that more resources like transmitters are needed for more targets or lower MSE requirements. The extensive random experiments provide the estimation performance and transmitter number for different targets number and estimation requirements, which can contribute to the evaluation on the traceability for given system sources and tracking task in fixed area in practical application.

In this work, resource allocation method for velocity estimation is studied. The future studies will concentrate on improving the joint location and velocity estimation performance. Moreover, the relation among target number, system resources, and performance requirements will be considered. Further, detection is the prerequisite for tracking, and a track-before-detect processing can actually help improve the detection probability, which should also be considered in the resource allocation problem of target detection and will be better to balance the resource allocation between detection and tracking tasks $[29,30]$. A resource allocation system with three tasks including a track-before-detect processing, target detection, and tracking should be taken into account.

\section{Appendix A}

Proof: According to (18), the optimal power $\mathbf{p}^{\text {opt }}$ and signal time $\mathbf{t}^{\mathrm{opt}}$ are collinear. We define a power vector $\hat{\mathbf{p}}$ and time vector $\hat{\mathbf{t}}$, satisfying
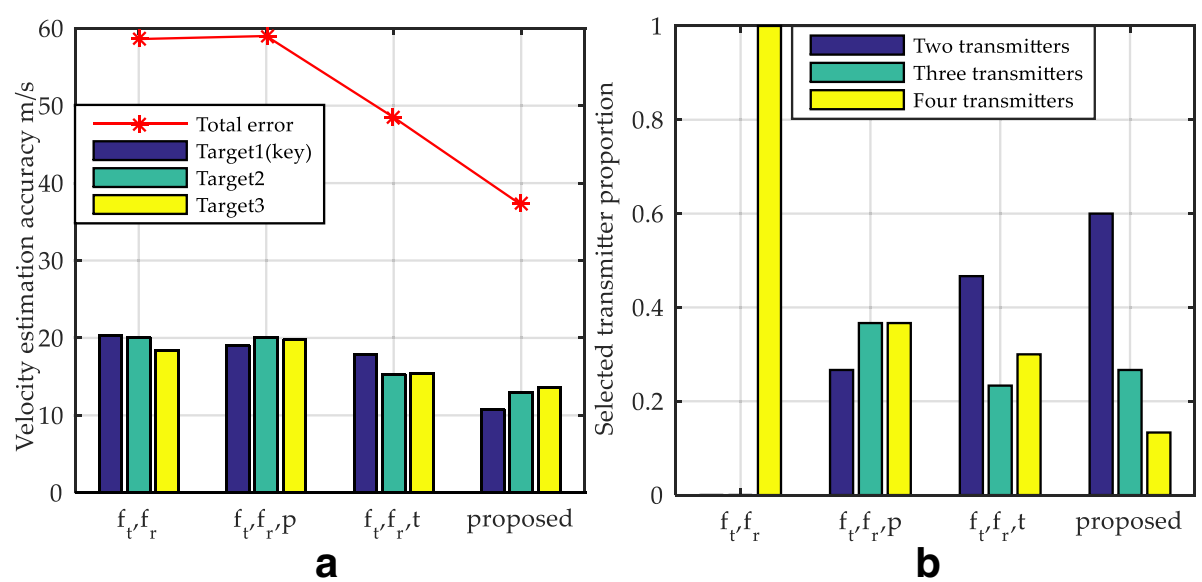

Fig. 7 Velocity estimation accuracy and transmitter number at random layout MSE $=\left[i n f, 20^{2}, 20^{2}\right] \mathrm{m}^{2} / \mathrm{s}^{2}$. a Velocity estimation accuracy $\mathbf{b}$ Transmitter proportion 


$$
\left\{\begin{array}{l}
\hat{p}_{i}=\frac{P_{\text {total }}}{\sum_{m=1}^{M}\left(p_{m}^{\mathrm{opt}}\right)^{\frac{1}{3}}\left(t_{m}^{\mathrm{opt}}\right)^{\frac{2}{3}}}\left(p_{i}^{\mathrm{opt}}\right)^{\frac{1}{3}}\left(t_{i}^{\mathrm{opt}}\right)^{\frac{2}{3}} \\
\hat{t}_{i}=\frac{T_{\text {total }}}{\sum_{m=1}^{M}\left(p_{m}^{\mathrm{opt}}\right)^{\frac{1}{3}}\left(t_{m}^{\mathrm{opt}}\right)^{\frac{2}{3}}}\left(p_{i}^{\mathrm{opt}}\right)^{\frac{1}{3}}\left(t_{i}^{\mathrm{opt}}\right)^{\frac{2}{3}}
\end{array}\right.
$$

Then,

$$
\operatorname{Tr}^{q}(\hat{\mathbf{p}}, \hat{\mathbf{t}})=\frac{\left[\sum_{m=1}^{M}\left(p_{m}^{\mathrm{opt}}\right)^{\frac{1}{3}}\left(t_{m}^{\mathrm{opt}}\right)^{\frac{2}{3}}\right]^{3}}{P_{\text {total }} T_{\text {Total }}^{2}} \operatorname{Tr}^{q}\left(\mathbf{p}^{\mathrm{opt}}, \mathbf{t}^{\mathrm{opt}}\right)
$$

By Holder inequality, $\quad\left[\sum_{m=1}^{M}\left(p_{m}^{\text {opt }}\right)^{\frac{1}{3}}\left(t_{m}^{\text {opt }}\right)^{\frac{2}{3}}\right]^{3} \leq$ $\left[\left(\mathbf{1}^{\mathrm{T}} \mathbf{p}^{\mathrm{opt}}\right)^{\frac{1}{3}}\left(\mathbf{1}^{\mathrm{T}} \mathbf{t}^{\mathrm{opt}}\right)^{\frac{2}{3}}\right]^{3}$ for $p_{m}^{\mathrm{opt}}, t_{m}^{\mathrm{opt}} \geq 0$. Due to the constraints of $\mathbf{1}^{\mathrm{T}} \mathbf{p}^{\text {opt }}=P_{\text {total }}$ and $\mathbf{1}^{\mathrm{T}} \mathbf{t}^{\text {opt }}=T_{\text {total, }}$ we can get

$$
\left[\sum_{m=1}^{M}\left(p_{m}^{\mathrm{opt}}\right)^{\frac{1}{3}}\left(t_{m}^{\mathrm{opt}}\right)^{\frac{2}{3}}\right]^{3} \leq P_{\text {total }} T_{\text {Total }}^{2}
$$

which is equivalent to

$$
\operatorname{Tr}^{q}(\hat{\mathbf{p}}, \hat{\mathbf{t}}) \leq \operatorname{Tr}^{q}\left(\mathbf{p}^{\text {opt }}, \mathbf{t}^{\text {opt }}\right)
$$

Consequently, we can obtain $\hat{\mathbf{p}}=\mathbf{p}^{\text {opt }}, \hat{\mathbf{t}}=\mathbf{t}^{\text {opt }}$. Therefore, for the joint transmitted power and signal time allocation, the optimal solution of (21) meets

$$
\mathbf{p}^{\text {opt }}=\frac{P_{\text {total }}}{T_{\text {Total }}} \mathbf{t}^{\text {opt }}
$$

\section{Appendix B}

Proof: According to (18), let $\mathbf{H}^{q} \mathbf{v}=\mathbf{y}$, applying (19), the equation $\lambda^{q} \times V^{q}\left(\mathbf{f}_{\mathrm{t}}, \mathbf{f}_{\mathrm{r}} \mathbf{p}, \mathbf{t}\right) \leq 1$ is equivalent to

$$
4 \lambda^{q} \boldsymbol{F}_{\mathrm{r}}^{\mathrm{T}} \mathbf{y} \leq \mathbf{y}^{\mathrm{T}} \boldsymbol{F}_{\mathrm{r}} \boldsymbol{F}_{\mathrm{r}}^{\mathrm{T}} \mathbf{y}-\mathbf{y}^{\mathrm{T}}\left(\boldsymbol{F}_{\mathrm{r}} \odot \boldsymbol{C}^{q}\right)\left(\boldsymbol{F}_{\mathrm{r}} \odot \boldsymbol{C}^{q}\right)^{\mathrm{T}} \mathbf{y}-\mathbf{y}^{\mathrm{T}}\left(\boldsymbol{F}_{\mathrm{r}} \odot \boldsymbol{S}^{q}\right)\left(\boldsymbol{F}_{\mathrm{r}} \odot \boldsymbol{S}^{q}\right)^{\mathrm{T}} \mathbf{y}
$$

After completing the square, we can get

$$
\begin{aligned}
\mathbf{y}^{\mathrm{T}} & \left(\boldsymbol{F}_{\mathrm{r}} \odot \boldsymbol{C}^{q}\right)\left(\boldsymbol{F}_{\mathrm{r}} \odot \boldsymbol{C}^{q}\right)^{\mathrm{T}} \mathbf{y} \\
& +\mathbf{y}^{\mathrm{T}}\left(\boldsymbol{F}_{\mathrm{r}} \odot \boldsymbol{S}^{q}\right)\left(\boldsymbol{F}_{\mathrm{r}} \odot \boldsymbol{S}^{q}\right)^{\mathrm{T}} \mathbf{y} \\
& +\left(2 \lambda^{q}\right)^{2} \leq\left(\boldsymbol{F}_{\mathrm{r}}{ }^{\mathrm{T}} \mathbf{y}-2 \lambda^{q}\right)^{2}
\end{aligned}
$$

Let $\mathbf{A}^{q}=\left[\mathbf{C}^{q}, \mathbf{S}^{q}, \mathbf{0}\right]^{\mathrm{T}}, \mathbf{b}=\left[0,0,2 \lambda^{q}\right]^{\mathrm{T}}$. Since $\mathbf{F}_{\mathrm{r}}^{\mathrm{T}} \mathbf{y}-2 \lambda^{q} \geq 0$, the inequality (35) can be equivalent to the SOCP.

$$
\left\|\left(\mathbf{F}_{\mathrm{r}} \odot \mathbf{A}^{q}\right) \mathbf{y}+\mathbf{b}\right\| \leq\left(\boldsymbol{F}_{\mathrm{r}}\right)^{\mathrm{T}} \mathbf{y}-2 \lambda^{q}
$$

\section{Abbreviations}

BCRB: Bayesian Cramer-Rao Bound; BIM: Bayesian information matrix; EKF: Extend Kalman filter; FIM: Fisher information matrix; LPI: Low probability of interception; MIMO: Multiple-input multiple-output; MSE: Mean square error;
SDP: Semi-definite programming; SNR: Signal to noise ratio; SOCP: Secondorder cone programming; SPCA: Sequential parameter convex approximation

\section{Acknowledgements}

The authors want to acknowledge the help of all the people who influenced the paper. Specifically, they want to acknowledge the anonymous reviewers for their reasonable comments.

\section{Ethic approval and consent to participate}

Not applicable.

\section{Funding}

The authors would like to acknowledge the support of the China National High Technology Major Project (Grant No. 2013ZX03006003-006) and the China Natural Science Foundation (Grant Nos. 61401469, 41301481, 61401513).

\section{Availability of data and materials}

The data is simulated as the provided parameters in Section 4.

\section{Authors' contributions}

XS proposed the three-step SOCP optimization method and carried out the numerical experiments. NZ proposed the worthy study problem and provide the paper organization. SY analyzed the system model and performance metric $\mathrm{HL}$ improved the writing. All authors read and approved the final manuscript. We appreciate the constructive comments received from the reviewers.

\section{Consent for publication}

Not applicable.

\section{Competing interests}

The authors declare that they have no competing interests.

\section{Publisher's Note}

Springer Nature remains neutral with regard to jurisdictional claims in published maps and institutional affiliations.

Received: 20 April 2018 Accepted: 1 October 2018

Published online: 19 October 2018

\section{References}

1. V.S. Chernyak, Fundamentals of multisite radar systems (Gordon and Breach Science Publishers Nv, America, 1998)

2. C.J. Baker, L. Hume, Netted radar sensing. IEEE Aerospace Electron. Syst. May 18, 3-6 (2003)

3. J. Li, P. Stoica, MIMO radar signal processing (Wiley-lEEE Press, New York, 2008)

4. D. Kalogerias, A. Petropulu, Matrix completion in collocated MIMO radar: recoverability, bounds \& theoretical guarantees. IEEE Trans. Signal Process 309-321, 62 (2014)

5. A. Haimovich, R. Blum, L. Cimini, MIMO radar with widely separated antennas. IEEE Mag Signal Process 25, 116-129 (2008)

6. D. Anastasios, L. Sangarapillai, C. Jonathon, Game theoretic analysis for MIMO radars with multiple targets. IEEE Trans. Aerosp Elect Syst 52, 2760-2774 (2016)

7. D. Deb, R. Bhattacharjee, A. Vengadarajan, Resource manager for MIMO radar (Proceedings of the IEEE Radar Conference, Washington DC, 2015), pp. 7175

8. H. Gao, J. Wang, X. Zhang, Resource allocation in MIMO radar with widely separated antennas for multi-target detection (Proceedings of the IEEE Radar Conference, 2014), pp. 1-6

9. M. Radmard, Antenna placement and power allocation optimization in MIMO detection. IEEE Trans. Aerosp Elect Syst 50, 1468-1478 (2014)

10. H. Chen, S. Ta, B. Sun, Cooperative game approach to power allocation for target tracking in distributed MIMO radar sensor networks. IEEE Sensors J. 15, 5423-5432 (2015)

11. P. Chavali, A. Nehorai, Scheduling and power allocation in a cognitive radar network for multiple-target tracking. IEEE Trans. Signal Proces 60, 715-729 (2012)

12. C. Shi, J. Zhou, F. Wang, LPI based resource management for target tracking in distributed radar network (IEEE Radar Conference, Philadelphia, 2016), pp. $822-826$ 
13. C. Shi, S. Salous, F. Wang, J. Zhou, Power allocation for target detection in radar networks based on low probability of intercept: a cooperative game theoretical strategy. Radio Sci 52(8), 1030-1045 (2017)

14. C. Shi, F. Wang, M. Sellathurai, et al., Power minimization based robust OFDM radar waveform design for radar and communication systems in coexistence. IEEE Trans. Signal Process. 99, 1 (2017)

15. C. Shi, F. Wang, M. Sellathurai, et al., LPI optimization framework for target tracking in radar network architectures using information-theoretic criteria. Int J Antenn Prop 21, 1-10 (2014)

16. H. Godrich, A. Haimovich, R.S. Blum, Target localization accuracy gain in MIMO radar-based systems. IEEE Trans. Inf Theory 56, 2783-2803 (2010)

17. H. Godrich, A.M. Haimovich, R.S. Blum, A MIMO radar system approach to target tracking (Proceedings of .Aslimar Conference Signals, Systems and Computer, 2009), pp. 1186-1190

18. N. Garcia, A.M. Haimovich, M. Coulon, Resource allocation in MIMO radar with multiple targets for non-coherent localization. IEEE Trans, Sign Process 62, 2656-2666 (2013)

19. Q. He, R. Blum, H. Godrich, Target velocity estimation and antenna placement for MIMO radar with widely separated antennas. IEEE J Sel Top Sign Process 4, 79-100 (2010)

20. H. Godrich, A.P. Petropulu, H.V. Poor, Sensor selection in distributed multiple-radar architectures for localization: a knapsack problem formulation. IEEE Trans. Sign Process 60, 247-260 (2012)

21. X. Song, N. Zheng, T. Bai, Resource allocation methods for multiple targets tracking in distributed MIMO radar systems. Int J Ant Prop 10, 1-12 (2017)

22. M. Xie, W. Yi, T. Kirubarajan, et al., Joint node selection and power allocation for multitarget tracking in decentralized radar networks. IEEE Trans. Signal Process. 99, 1 (2016)

23. Y. Shen, W. Dai, M. Win, Power optimization for network localization. IEEE/ ACM Trans. Networking 22, 1337-1350 (2014)

24. B. Ma, H. Chen, B. Sun, A joint method of antenna selection and power allocation for localization in MIMO radar sensor networks. IEEE Int Conf Sign Process 18, 1089-7798 (2014)

25. P. Tichavsky, C.H. Muravchik, A. Nehorai, Posterior Cramer-Rao bounds for discrete-time nonlinear filtering. IEEE Trans Sign Process 46, 1386-1396 (1998)

26. S.M. Kay, Fundementals of statiscal signal processing: estimation theory, 1st edn. (Parentice Hall PTR, New Jersey, 1993)

27. S. Boyd, L. Vandenberghe, Convex optimization (Cambridge University, Cambridge. UK, 2004), pp. 67-127

28. S.S. Rao, Engineering optimization: theory and practice, 3rd edn. (Wiley, New York, 1996), pp. 383-425

29. Ehlers, F., Orlando, D., Ricci, G. A Batch tracking algorithm for multi-static sonars. IET Radar Sonar Navig. 6: 746-752(2012)

30. D. Orlando, G. Ricci, Y. Bar-Shalom, Track-before-detect algorithms for targets with kinematic constraints. IEEE Trans. Aerosp. Electron. Syst. 47, 1837-1849 (2011)

\section{Submit your manuscript to a SpringerOpen ${ }^{\circ}$ journal and benefit from:}

- Convenient online submission

- Rigorous peer review

- Open access: articles freely available online

High visibility within the field

- Retaining the copyright to your article

Submit your next manuscript at $\boldsymbol{\nabla}$ springeropen.com 